\title{
A study on the actual conditions associated with the presence of Acinetobacter sp. in a hospital waiting room
}

\author{
Akane Odagiri ${ }^{*}$, U Yanagi ${ }^{2}$, Miyoko Endo ${ }^{3}$ and Hisato $\mathrm{Oda}^{4}$ \\ ${ }^{1,2}$ School of Architecture, Kogakuin University, Japan \\ ${ }^{3}$ Biomedical Science Association, Japan \\ ${ }^{4}$ SINKO Industries Ltd., Japan
}

\begin{abstract}
In recent years, nosocomial infection, including that by multiple-drug-resistant Acinetobacter (MDRA), has become an increasing concern in Japan. Typically, Acinetobacter inhabits soil and river water and is usually harmless to humans. However, bacteria of this genus can become pathogens in inpatients with weakened immune systems, resulting in opportunistic infection. Currently, hospital infection by MDRA is spreading not only in Japan, but also worldwide. This is a very serious problem, and little is known about effective countermeasures. The present study sought to examine the microbiome status in a hospital visitor waiting room as a first step in establishing countermeasures for use against Acinetobacter. Specifically, we performed a so-called metagenomic analysis to directly analyze DNA collected from the environment, without an intervening cultivation step. Indoor airborne bacteria and surface bacteria were sampled and analyzed. The results show that in a visitor waiting room, the top-ten most-abundant genera included (in decreasing order) Acinetobacter, Streptococcus, Prevotella, Pseudomonas, Bifidobacterium, Fusobacterium, Neisseia, Porphyromonas, Leptotrichia, and Haemophilus. Thus, Acinetobacter constituted the mostdetected genus and was present in all collected samples. Furthermore, we assessed the bactericidal effect of ultraviolet C (UVC) against Acinetobacter sp. 7206, a hospital isolate. A UVC dose of $4.0 \mathrm{sec} \cdot \mathrm{mW} / \mathrm{cm}^{2}$ was required to kill the $99.9 \%$ Acinetobacter sp. 7206 on solid culture medium.
\end{abstract}

\section{Introduction}

In recent years, nosocomial infection, including that by multiple-drug-resistant Acinetobacter (MDRA), has become an increasing concern in Japan. Notably, MDRA and similar bacteria were detected in 15 patients in the Kagoshima University Hospital ICU (intensive care unit) in the summer of 2018. Four of these 15 inpatients died during their hospitalizations, and three of these four mortalities were attributed to MDRA. The hospital administration subsequently revealed that MDRA was recovered from three of sixteen mattresses in this ICU.

Commonly, members of the genus Acinetobacter inhabit soil and river water and are usually harmless to humans. However, these bacteria can become pathogens in inpatients with weakened immune systems, resulting in opportunistic infection. However, antibiotics that are typically used for the medical treatment of Acinetobacter infection have reduced efficacy against drug-resistant Acinetobacter in Japan. Currently, nosocomial infection caused by MDRA is spreading not only in Japan but also worldwide. This is very serious problem, and little is known about effective countermeasures.

The present study sought to examine the microbiome status in a hospital visitor waiting room as a first step in establishing countermeasures against Acinetobacter. Specifically, we performed a so-called metagenomic analysis to directly analyze DNA collected from the environment without an intervening cultivation step. Furthermore, we conducted an experiment on the bactericidal effect of ultraviolet C (UVC) as a treatment against Acinetobacter sp. 7206, a hospital isolate.

\section{Materials and methods}

\subsection{The outline of the hospital}

The investigation site was located at a 778bed hospital that has a total floor area of $56,635 \mathrm{~m}^{2}$. The assessments described in this report were performed in the visitor waiting room $\left(74 \mathrm{~m}^{2}\right.$ floor area) of neurological diseases. An air-handling unit was installed in the waiting room.

\subsection{Field measurement}

Field measurement was carried out from January 28 to 31 , 2018. The measured parameters included temperature, relative humidity $(\mathrm{RH})$, carbon dioxide $\left(\mathrm{CO}_{2}\right)$ concentration, and airborne and surface microbial populations. Five measurement locations were selected: the waiting room on the second floor, return and supply air in the air-handling unit, and outdoors. The following

Corresponding author: yanagi@cc.kogakun.ac.jp 
methods were used to measure the parameters. Air temperature, $\mathrm{RH}$, and $\mathrm{CO}_{2}$ concentrations were recorded once per min using a data logger (TR-76Ui) during measurement period.

Airborne microbes were collected using an air pump (Air Check XR5000) equipped with a filter (PTFE

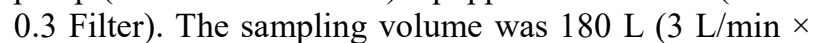
$60 \mathrm{~min})$.

After measurement of the temperature, $\mathrm{RH}$, and $\mathrm{CO}_{2}$ level, surface microbes were collected. Surface microbes were recovered by wiping using a swab kit. Adhering microbes were collected by swabbing (separately) a chair and a doorknob in the waiting room, as well as the main filter and pre-filter in the air conditioner. For each item, an area totaling $100 \mathrm{~cm}^{2}$ was swabbed, including $10 \mathrm{~cm} \times 10 \mathrm{~cm}$ surfaces of the chair, the main filter, and the pre-filter, and an $8 \mathrm{~cm} \times 12.5 \mathrm{~cm}$ surface of the doorknob before and after UVC operation.

\subsection{Sterilization by UVC}

The experiment testing sterilization of a purified isolate of Acinetobacter by UVC was performed under the following conditions.

The test bacterium was Acinetobacter sp. 7206, a hospital isolate, plated on solid culture medium at 1600 colony-forming units (cfu) per plate. The UVC intensity at the surface of the culture medium was $0.1 \mathrm{~mW} / \mathrm{cm}^{2}$; irradiation times were $0,20,25,30,35,40$, or $50 \mathrm{sec}$.

\subsection{DNA extraction, amplification, sequencing}

\section{(1) DNA extraction}

Each sample was placed in a sterilization bag together with double-distilled water. After processing the samples using a Stomacher 80 Biomaster, the extract was transferred to a test tube and centrifuged (KUBOTA3700). The centrifuged extract was divided into bacterial and viral components, but only bacterial DNA was purified and analyzed in the present study. Bacterial DNA was purified using the NucleoSpin Tissue Kit (740952.50).

(2) DNA amplification, Sequencing, Data analysis

For details of DNA extraction, amplification and sequencing by next generation sequencer, please refer to the previous report.(Yanagi, ex al 2018)

\section{Results}

\subsection{Environment of the waiting room}

Air temperature and $\mathrm{RH}$ in the waiting room were $26 \pm 1^{\circ} \mathrm{C}$ and $41 \pm 3 \%$, respectively. These $\mathrm{RH}$ did not satisfy the Healthcare Engineering Association of Japan standards (temperature: 26 to $27^{\circ} \mathrm{C}, \mathrm{RH}: 50$ to $60 \%$ ). $\mathrm{CO}_{2}$ concentration was $697 \pm 35 \mathrm{ppm}$, a value that complied with relevant standards, such as the Japanese 'Law for Environmental Health in Buildings'.

\subsection{Detected bacteria}

Table 1 shows the number of detected bacteria by phylum, class, order, family, and genus for all prokaryotes with a relative abundance exceeding $1.0 \%$ of the total. Compared to outdoor air, a larger number of phylums, classes, orders, families, and genuses were detected in the indoor environment. Notably, multiple phylums, classes, orders, families, and genuses were detected from the upstream and downstream of a coil. Similarly, multiple phylums, classes, orders, families, and genuses were detected from the tested surfaces.

Fig. 1 shows reads of genera that ranked in the top 10 for abundance (excepting reads from outdoor air). The metagenomes included bacteria of the genera Acinetobacter, Streptococcus, Prevotella, Pseudomonas, Bifidobacterium, Fusobacterium, Neisseria, Porphyromonas, Leptotrichia, and Haemophilus. Notably, the most frequently detected genus was Acinetobacter, which was detected in all samples. Acinetobacter inhabits soil and river water and usually is harmless to humans. Acinetobacter previously has been detected only rarely in indoor environments such as offices. These observations have been used to suggest that the genus Acinetobacter exists primarily in hospital settings.

To date, 17 species (including 15 genomic species) have been classified as members of the genus Acinetobacter based on metagenomic analyses. Among these species, three (Acinetobacter baumannii, Acinetobacter genomic species 13TU, and Acinetobacter genomic species 3 ) are considered MDRA.

Table 2 shows detection of species and number of reads in our analysis. Names marked in red represent known strict pathogens; those marked in orange represent known opportunistic pathogens. Our metagenomic analysis did not detect A. baumannii, Acinetobacter genomic species 13TU, or Acinetobacter genomic species 3 . However, only $578(3.3 \%)$ of the reads that were assigned to the genus Acinetobacter could be identified at the species level. Among the bacteria of known species, the following opportunistic and strict pathogens were detected.

Table 1. Number of detected bacteria by phylum, class, order, family, and genus

\begin{tabular}{|c|c|c|c|c|c|c|}
\hline & & Phylum & Class & Orde & Family & Genus \\
\hline \multirow{4}{*}{ Air } & $\mathrm{OA}$ & 12 & 32 & 32 & 44 & 69 \\
\hline & RA & 11 & 33 & 29 & 43 & 80 \\
\hline & Upstream of Coil fin & 12 & 20 & 31 & 41 & 31 \\
\hline & $\begin{array}{l}\text { Downstream of Coil } \\
\text { fin }\end{array}$ & 10 & 17 & 28 & 40 & 33 \\
\hline \multirow{5}{*}{ Surface } & Chair & 11 & 16 & 24 & 32 & 42 \\
\hline & Doorknob & 9 & 17 & 25 & 34 & 24 \\
\hline & Main filter & 6 & 14 & 21 & 31 & 25 \\
\hline & Prefilter & 8 & 13 & 21 & 23 & 15 \\
\hline & Coil fin & 9 & 15 & 22 & 32 & 23 \\
\hline
\end{tabular}

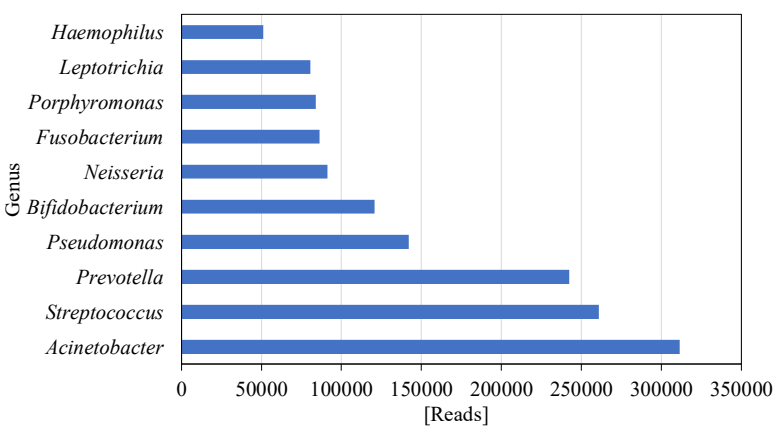

Fig. 1. Most abundant ( $>1 \%$ of sequences) genera 
Acinetobacter lwoffii: This species has been detected primarily in humans, where the organism has been identified as an opportunistic pathogen in meningitis cases, especially in post-operative meningitis. A. lwoffii was detected all samples in the present study.

Acinetobacter segnis is a pathogen associated with infectious diseases of the intraoral and digestive systems, and has been recovered from cases of periodontitis, cholecystitis, appendicitis, and pancreatic abscess, and as a causative bacterium in primary sepsis. In the present study, A. segnis was detected primarily from the main air filter and in both front and rear filter spaces.

Bacteroides uniformis typically coexists with humans but serves as an opportunistic pathogen within the peritoneal cavity. In the present study, B. uniformis was detected in large amounts from RA on a single day (January 30, 2018).

Bacteroides fragilis is one of the predominant bacteria constituting the human gastrointestinal microbiome and is present from the human oral cavity through the intestine. This organism has low pathogenicity in general but does cause disease when the body's resistance is decreased. In the present study, $B$. fragilis was detected in the RA and on the doorknob.

Capnocytophaga ochracea is present in human oral and non-oral cavities and is related to periodontitis in both children and adults. This organism rarely causes serious infections, but has been recovered from cases of endocarditis, endometriosis, osteomyelitis, abscess, peritonitis, and keratitis. In the present study, $C$. ochracea was detected in large amounts from the chair on a single day (January 30, 2018).

Haemophilus influenzae is a bacterium that is normally present in the upper respiratory tract of humans and is a causative agent of various infectious diseases associated with the respiratory system. In the present study, $H$. influenzae was detected primarily from the main air filter and in both front and rear filter spaces.

Neisseria subflava is an opportunistic pathogen that is commonly observed in the human oral and respiratory tract. In the present study, $N$. subflava was detected on the majority of surveyed surfaces.

Prevotella nigrescens is an opportunistic pathogen that is commonly detected in the human oral and respiratory tract. In the present study, this organism was detected in large amounts from the rear of the filter space on a single day (January 30, 2018).

Rothia mucilaginosa is present in the human oral cavity but in rare cases serves as a causative organism for infectious diseases such as bacteremia, meningitis, and pneumonia. In the present study, this species was detected in large amounts on all surveyed surfaces.

Streptococcus anginosus has been detected from various human sites and can cause infections and abscesses.

Three UV lamps were set forward of a coil fin on the air-handling unit. A clear effect with UVC irradiation was not detected at the level of the number of metagenomic reads. This analysis is considered an example of the potential use situation for sterilization in the waiting room, given that the level of bacterial contamination in the RA would be changing at every moment.

\subsection{Principal coordinate analysis (PCoA)}

Table 2. Detected species

\begin{tabular}{|c|c|c|c|c|c|c|c|c|c|c|c|c|c|c|c|c|c|c|}
\hline & \multicolumn{5}{|c|}{$2018 / 1 / 28$} & \multicolumn{13}{|c|}{$2018 / 1 / 29$} \\
\hline UVLamp & & & & & & before & after & before & after & before & after & before & after & & & & & \\
\hline Sampling point & MF & PF & $\mathrm{CF}$ & $\mathrm{Ch}$ & $\mathrm{Dk}$ & FIL f & FIL $f$ & FIL r & FIL $r$ & RA & RA & $\mathrm{OA}$ & $\mathrm{OA}$ & MF & $\mathrm{PF}$ & $\mathrm{CF}$ & $\mathrm{Ch}$ & Dk \\
\hline Acinetobacter lwoffii & 97 & 0 & 18 & 406 & 51 & 77 & 8 & 113 & 26 & 80 & 141 & 86 & 8 & 55 & 1 & 62 & 197 & 56 \\
\hline Acinetobacter rhizosphaerae & & 0 & 0 & & 0 & & 0 & 0 & 0 & & 0 & 7 & 0 & 0 & & 0 & & 4 \\
\hline Aggregatibacter segnis & & 0 & & & & & & & & & & & 0 & 128 & 0 & 0 & & \\
\hline Akkermansia muciniphila & & 0 & 0 & 2 & 3 & 0 & & 2 & & & & & 0 & 0 & & 0 & & 0 \\
\hline Anoxvbacillus kestanbolensis & & 0 & 1 & 60 & 50 & 0 & 0 & 0 & 0 & 233 & 98 & 0 & 10 & 25 & & 26 & & \\
\hline Bacillus humi & 0 & 0 & 0 & 0 & 0 & 0 & 0 & 0 & 0 & 0 & 0 & 0 & 76 & 0 & 0 & 106 & 0 & 3 \\
\hline Bacteroides uniformis & 0 & 0 & 0 & 0 & 0 & 0 & 0 & 0 & 0 & 0 & 0 & 0 & 0 & 0 & & 0 & 0 & 0 \\
\hline Bacteroides fragilis & 0 & 0 & 0 & 0 & 0 & 0 & 0 & 0 & 0 & 859 & 13 & 0 & 0 & 72 & & 0 & 0 & 199 \\
\hline Bifidobacterium adolescentis & 29 & 0 & 236 & 359 & 64 & 18 & 2 & 32 & 33 & & 123 & 359 & 854 & 131 & & 32 & 44 & 29 \\
\hline Brachybacterium conglomeratum & 1 & 0 & 0 & 0 & 3 & 0 & 0 & 0 & 0 & 0 & 0 & 1 & 2 & 1 & & $\begin{array}{ll}0 & 2835 \\
\end{array}$ & 0 & 0 \\
\hline Capnocytophaga ochracea & 0 & 0 & 0 & 0 & 0 & 0 & 0 & 5 & 0 & 0 & 0 & 0 & 1 & 0 & 0 & \begin{tabular}{l|r}
0 & 0 \\
\end{tabular} & 0 & 0 \\
\hline Corynebacterium durum & 1 & 0 & 941 & 1 & 0 & 0 & 0 & 2 & 0 & 0 & 0 & 0 & 0 & 0 & & 0 & 16 & 98 \\
\hline Deinococcus geothermalis & 380 & 0 & 0 & 0 & 0 & 0 & 0 & 0 & 0 & 0 & 0 & 1681 & 0 & 0 & & 0 & 0 & 0 \\
\hline Eggerthella lenta & 0 & 0 & 0 & 0 & 0 & 0 & 0 & 0 & $\frac{0}{0}$ & 0 & 0 & 0 & 0 & 699 & & 41 & 0 & \\
\hline Enterococcus cecorum & 0 & 0 & 1 & 0 & 0 & 0 & 1 & 0 & 235 & 0 & 2 & 0 & 0 & 0 & & 0 & 35 & \\
\hline Faecalibacterium prausnitzii & 4 & 0 & 0 & 0 & 3 & 0 & 0 & 0 & 0 & 0 & 46 & 0 & 0 & 0 & & 0 & & 49 \\
\hline Geodermatophilus obscurus & 0 & 0 & 22 & 0 & 0 & 0 & 0 & 0 & 0 & 0 & 0 & 0 & 0 & 0 & & 0 & 0 & 0 \\
\hline Haemophilus parainfluenzae & 19 & 0 & 2 & 91 & 8 & 13 & 0 & 0 & 0 & 8 & 163 & 1 & & 163 & & 1 & & 55 \\
\hline Haemophilus influenzae & & & & & & & & & & & & & & & & & & \\
\hline Kocuria rhizophila & & 3 & 20 & 195 & 2 & 74 & 0 & 16 & 0 & 0 & 45 & 2 & 643 & 0 & & 0 & 34 & 50 \\
\hline Kocuria palustris & 0 & 0 & 0 & 79 & 37 & & 0 & 0 & 6 & 0 & 2 & 1 & 157 & 0 & & 0 & 10 & 0 \\
\hline Lactobacillus iners & 164 & 0 & 1 & 0 & 2 & 992 & 0 & 0 & 0 & 0 & 1 & 0 & 0 & 298 & & 0 & 1 & 0 \\
\hline Lysobacter brunescens & & 0 & 0 & 0 & 0 & 0 & 1078 & 0 & 0 & 0 & 0 & 0 & 17 & 0 & & 0 & 0 & \\
\hline Methylobacterium organophilum & 0 & 0 & 92 & 0 & 0 & 0 & 0 & 0 & 0 & 0 & 86 & 0 & & 0 & & 0 & 0 & 0 \\
\hline Methvlotenera mobilis & & 0 & 19 & 0 & 0 & 0 & 0 & 0 & 0 & 0 & 0 & 0 & 0 & 1 & 3841 & & 0 & 0 \\
\hline Neisseria subflava & 9 & 0 & 8 & 23 & 51 & 2 & 63 & 314 & 39 & 26 & 53 & & 42 & 51 & & 26 & 35 & 16 \\
\hline Parabacteroides distasonis & 0 & 0 & 0 & 0 & 0 & 0 & 0 & 0 & 0 & 0 & 0 & 0 & 0 & 0 & & 0 & 0 & 0 \\
\hline Paracoccus aminovorans & & 0 & 21 & 0 & 10 & 0 & & 1411 & 0 & 0 & 0 & 0 & 0 & 0 & & 142 & 0 & \\
\hline Porphyromonas endodontalis & 0 & 0 & 0 & 0 & 0 & 0 & 0 & 0 & 116 & 0 & 24 & & 0 & 37 & 0 & 0 & 0 & 0 \\
\hline Prevotella melaninogenica & 1943 & 8 & 4431 & 2270 & 1234 & 10 & 17072 & 10120 & 12804 & 5246 & 4843 & 15530 & 7354 & 4306 & 19 & 4540 & 10258 & 4414 \\
\hline Prevotella nanceiensis & 2838 & 0 & 1250 & 159 & 751 & 2914 & & 252 & $\begin{aligned} 1 \\
\end{aligned}$ & 2 & 808 & 36 & 357 & 87 & & 2775 & 7420 & 2008 \\
\hline Prevotella nigrescens & & 0 & 0 & 0 & 0 & 0 & 0 & 1388 & 0 & 0 & 30 & 0 & 0 & 0 & & 0 & 0 & \\
\hline Prevotella copri & 364 & 0 & 7 & 52 & 89 & 0 & 6 & 0 & 2 & 252 & 1 & 0 & 0 & 3 & & 0 & 14 & \\
\hline Prevotella intermedia & 0 & 0 & 0 & 0 & 0 & 0 & 0 & 0 & 0 & 0 & 0 & 0 & 0 & 0 & & 0 & 0 & 2 \\
\hline Propionibacterium acnes & 419 & 7 & 192 & 23 & 47 & 8 & 21 & 24 & 325 & 5 & 425 & 39 & 84 & 49 & & 261 & 197 & 420 \\
\hline Pseudomonas viridiflava & 0 & 0 & & 0 & 0 & 0 & 532 & 2 & 1 & 0 & 0 & 0 & 0 & 0 & & 0 & 0 & 0 \\
\hline Psvchrobacter sanguinis & & 0 & & & & & & 0 & 0 & 0 & 0 & 0 & 0 & 149 & & 0 & 0 & 32 \\
\hline Roseomonas mucosa & 10 & 2 & 1171 & 2 & 0 & 1358 & & 1 & 1 & 1 & 566 & 650 & 0 & 155 & & 0 & 7 & 152 \\
\hline Rothia mucilaginosa & & & & & & & & & & & & & & & & & & \\
\hline Rothia aeria & & 0 & 24 & 105 & 0 & 1 & 178 & 449 & & 1863 & 30 & 290 & & 60 & & 0 & 0 & 25 \\
\hline Rothia dentocariosa & & 0 & 76 & 182 & 1 & 0 & 1 & 0 & 0 & 30 & 72 & 11 & 2 & 21 & 0 & 112 & 15 & 69 \\
\hline Selenomonas noxia & 2 & 0 & 0 & 0 & 0 & 0 & 0 & 0 & 0 & 0 & 0 & 0 & 0 & 1 & 0 & 0 & 32 & 54 \\
\hline Staphylococcus epidermidis & 0 & 0 & 0 & 0 & 14 & 0 & 1 & 0 & 0 & 0 & 1 & 0 & 408 & 0 & 0 & 0 & 0 & 0 \\
\hline Streptococcus anginosus & & & & & & & & & & & & & & & & & & \\
\hline Veillonella parvula & 804 & 0 & 992 & 543 & 541 & 36 & 90 & 878 & 96 & 389 & 380 & 349 & & 18 & & 1168 & 1291 & 266 \\
\hline Veillonella dispar & 4 & 0 & & 2 & 2 & 0 & 0 & 4 & 0 & 0 & 2 & 2 & 0 & 0 & 0 & 0 & 1 & 2 \\
\hline
\end{tabular}


Table 2. Detected species, continue

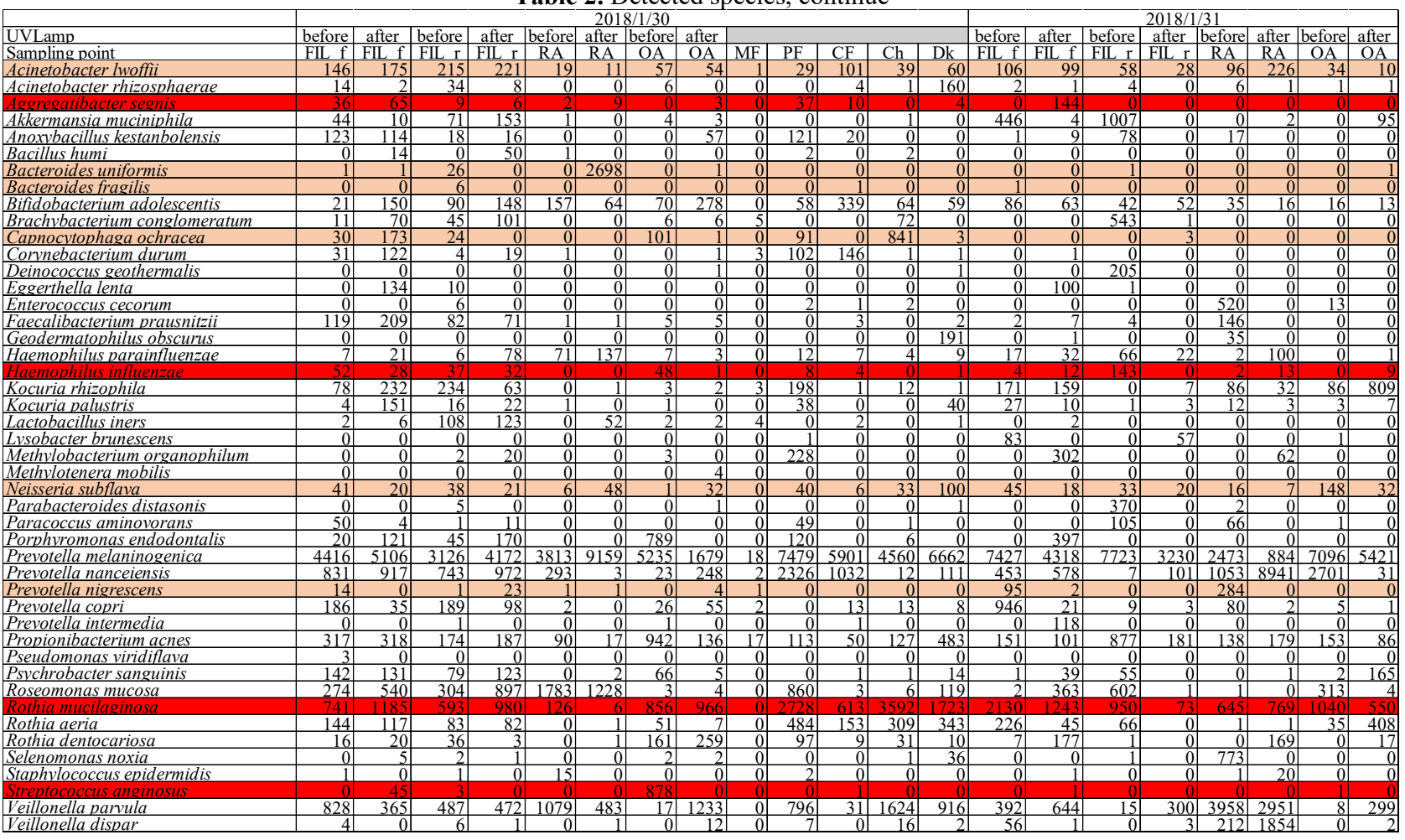

Fig. 3 shows the results obtained from principal coordinate analysis (PCoA). Most of the flora plotted to near positions. However, the flora of $1 / 28 \mathrm{PF}$ (Prefilter), $1 / 29 \mathrm{PF}$, and $1 / 30 \mathrm{MF}$ (Main filter) plotted as points distinct from the other specimens. These three samples contain flora extracted from the air filter of the airhandling unit, and therefore represent airborne microbes that adhered to the filter surface.
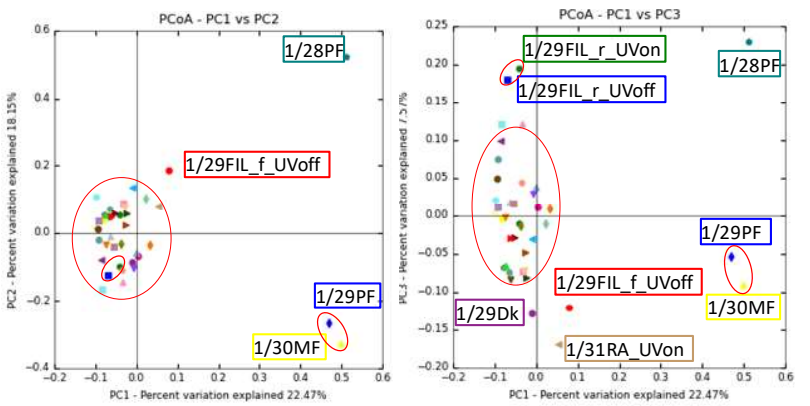

Fig. 3. The results obtained from $\underline{P C o A}$.

Table 3. The survival count (cfu) of UVC-irradiated Acinetobacter sp. 7206

\begin{tabular}{c|ccc|c}
\hline Elapsed time & Test1 & Test2 & Test3 & Ave \\
\hline $0 \mathrm{~s}$ & 1600 & 1600 & 1600 & 1,600 \\
$20 \mathrm{~s}$ & 138 & 173 & 286 & 199 \\
$25 \mathrm{~s}$ & 126 & 99 & 96 & 107 \\
$30 \mathrm{~s}$ & 66 & 52 & 73 & 64 \\
$35 \mathrm{~s}$ & 7 & 25 & 40 & 24 \\
$40 \mathrm{~s}$ & 0 & 0 & 1 & 0.3 \\
$50 \mathrm{~s}$ & 0 & 0 & 0 & 0 \\
\hline
\end{tabular}

\subsection{Bactericidal effect of UVC on Acinetobacter}

Table 3 shows the results obtained in an assessment of the bactericidal activity of UVC on Acinetobacter sp. 7206 in the laboratory. This table shows acinetobacterial survival as a function of irradiation time. According to Table 6, 99.98\% of Acinetobacter sp. 7206 strain were killed after $40 \mathrm{sec}$ of exposure, corresponding to a net UVC dose of $4 \mathrm{sec} \cdot \mathrm{mw} / \mathrm{cm}^{2}$.

\section{Conclusions}

In this study, it was found that many pathogens were detected in a hospital waiting room. Additionally, an insitu test was performed by installing UV lamps in the air-conditioning equipment of the hospital waiting room, and the effect of UVC irradiation also was assessed in an experiment in which Acinetobacter sp. 7206 was directly irradiated in the laboratory. The lab experiment demonstrated that a UVC dose of $4 \mathrm{sec} \cdot \mathrm{mw} / \mathrm{cm}^{2}$ achieved sterilization ( $>3-\log$ decrease in $\mathrm{cfu})$. On the other hand, As the object hospital was in use and the measurements had strong restrictions, a clear effect of UVC was not found in the in-situ test.

\section{References}

1. U Yanagi, Kato Shinsuke, Hatanaka Miku: Establishing the monitoring method of respiratory system pathogen in built environment -Part 1 the study outline, sampling and DNA analysis method, proceedings of Architectural Institute in Japan annual meeting, pp.859-862 2018

2. The Ministry Health, Labour and welfare, https://www.mhlw.go.jp/stf/seisakunitsuite/bunya/0 00013264.htm (access date; 20, Oct., 2018)

3. U Yanagi: Ultraviolet rays sterilization - past, present and future, Journal of Air Purification, Vol.51, No.3, pp.4-9 2013 